\title{
A Game-Theoretic Analysis of the Adversarial Boyd-Kuramoto Model
}

\author{
Antonin Demazy ${ }^{1}$, Alexander Kalloniatis ${ }^{2}$, and Tansu Alpcan ${ }^{1}$ \\ 1 Department of Electrical and Electronic Engineering, \\ The University of Melbourne, VIC, Australia. \\ tansu.alpcan@unimelb.edu.au \\ 2 Defence Science and Technology Group, \\ Canberra, ACT, Australia. \\ alex.kalloniatisedst.defence.gov.au
}

\begin{abstract}
The "Boyd", also known as the "OODA loop" models the cyclic decision processes of individuals and organisations in a variety of adversarial situations. Combined with the Kuramoto model, which provides a mathematical foundation for describing the behaviour of a set of coupled or networked oscillators, the Boyd-Kuramoto model captures strategic (cyclic) decision making in competitive environments.

This paper presents a novel game-theoretic approach to the Boyd-Kuramoto dynamical model in complex and networked systems. A two-player, Red versus Blue, strategic (non-cooperative) game is defined to describe the competitive interactions and individual decision cycles of Red and Blue agent populations. We study the model analytically in the regime of near phase synchrony where linearisation approximations are possible. We find that we can solve for the Nash equilibrium of the game in closed form, and that it only depends on the parameters defining the fixed point of the dynamical system. A detailed numerical analysis of the finite version of the game investigates the behaviour of the underlying networked Kuramoto oscillators and yields a unique, dominant Nash equilibrium solution. The obtained Nash equilibrium is further studied analytically in a region where the underlying Boyd-Kuramoto dynamics are stable. The result suggests that only the fixed point of the dynamical system plays a role, consist with the analytical solution. Finally, the impact of other variations of the Boyd-Kuramoto parameters on the game outcomes are studied numerically, confirming the observations from fixed point approaches. It is observed that many parameters of the Kuramoto model affect the NE solution of the current game formulation much less than initially stipulated, arguably due to the time-scale separation between the underlying Kuramoto model and the static game formulation.
\end{abstract}

\section{Introduction}

The Boyd or OODA loop is a cyclic decision process consisting of the following four stages: Observe, Orient, Decide, and Act [4]. It models the decision cycle of individuals and organisations in a variety of adversarial and competitive situations. It is a wellknown concept in business, litigation, and law enforcement. For example, the model can be used to capture the competition (for a pool of customers) between two companies 
in their innovation cycles as they bring new products to the market. The OODA loop is also used widely in military strategy, and operational Command and Control (C2), far from the air-to-air combat context in which it was originally derived.

The OODA loop matches the fundamental paradigms of system and control theory. The "Observe" stage involves making observations about the environment and collecting data by an Observer. The "Orient" stage may process the observed information in light of the identified models and past experiences. In the "Decide" stage, available actions are evaluated based on some performance criteria derived from utility, optimisation, or game theories. "Orient" and "Decide" can be mapped to a Controller in control theory. Finally, an action is taken in the "Act" stage, which corresponds to the behaviour of Actuators in systems. A similar analogy can also be made to strategic decision making by companies or organisations. Competitive firms observe the market trends and orient themselves accordingly; decide on their strategic investments, e.g. for innovation; and act to bring new products and services to the market to get a higher share.

The Kuramoto model [9] provides a mathematical foundation for describing the behaviour of a set of coupled or networked oscillators and their synchronisation characteristics. In this context, each oscillator corresponds to the decision cycle of an individual or part of an organisation. The Kuramoto model has been widely used to analyse complex systems in a variety of domains [1]. A novel application of Kuramoto oscillators to Boyd-OODA cycles in adversarial interactions between two populations, e.g. Red versus Blue, has been investigated in [7]. Specifically, the internal synchronisation of individual OODA oscillators within the (Red and Blue) populations as well as the average phase difference ("lags" or frustrations) between the populations have been studied in detail [6]. Such a model is an application of certain multi-graph frustrated generalisation of the Kuramoto model, as studied in works such as [5]. This combined Boyd-Kuramoto model is immediately applicable to competitive decisions as it captures the complex cyclic decision processes in adversarial populations of networked heterogeneous agents. Going back to the example of two competitive firms, the average phase difference may represent how much ahead one firm is against the other in the innovation cycle.

Game Theory studies multi-agent decision making with versatile quantitative methods. As a special case, security games have been used to successfully model the decision processes in adversarial situations [2]. The inherently adversarial nature of the interaction between Blue and Red populations in commercial competition or strategic $\mathrm{C} 2$ decision making immediately motivates a game-theoretic approach to the Boyd-Kuramoto model.

This paper applies the mathematical and conceptual framework of security games to the Boyd-Kuramoto model of networked decision cycles (oscillators) in Blue and Red populations within the adversarial/competitive context. As a starting point, a novel Boyd-Kuramoto Game is introduced. In this 2-Player, nonzero-sum strategic (non-cooperative) game, the Blue and Red players aim to gain a phase (lag) advantage over their opponent based on the average phase of their own agent populations, while achieving internal synchronisation of their Boyd-OODA oscillators. Kuramoto's order parameters of the player populations and the average phase difference between them provide the main cri- 


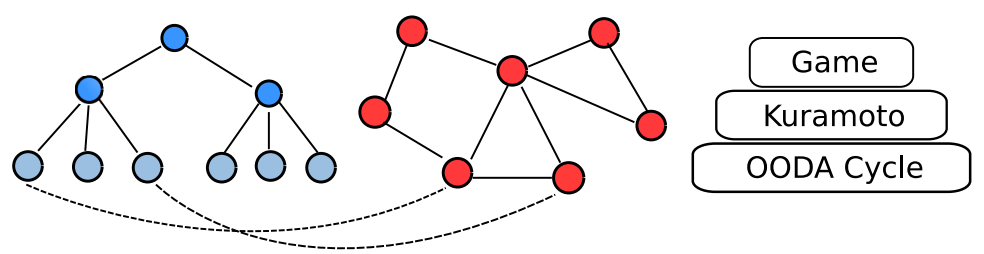

Fig. 1: A conceptual diagram of the Boyd-Kuramoto Game.

teria in modelling the player utility functions. This three-layer Boyd-Kuramoto Game model (Figure 1) is the first of its kind to the best knowledge of the authors.

The contributions of this paper include:

- Development of a novel game-theoretic model of Boyd-Kuramoto networked oscillators in complex $\mathrm{C} 2$ systems, which describes the strategic decision-making processes of Red vs Blue populations.

- A detailed numerical analysis of the game investigating the stable behaviour of the underlying networked Kuramoto oscillators, and computing the Nash Equilibrium solutions.

- A numerical study of the impact of Boyd-Kuramoto parameters on the game outcomes.

- An analytic description of the Nash Equilibrium solution after linearisation of the dynamical equations.

The rest of the paper is organised as follows. The next section presents the main components of the Boyd-Kuramoto Model. Section 3 introduces a specific game-theoretic formulation. A numerical analysis of the game is presented in Section 4. The sensitivity of the game solutions to various parameters is discussed in Section 6. Analytic solution is pursued in Section 5. The paper concludes with remarks and a discussion on future directions in Section 7.

\section{Boyd-Kuramoto Model for Complex Competitive Systems}

We consider a deterministic Blue versus Red networked Kuramoto oscillator model to describe complex, adversarial, and competitive interactions using Boyd (OODA) cycles in both Blue and Red agent populations. Let $\mathcal{B}=\{1, \ldots, N\}$ and $\mathcal{R}=\{1, \ldots, M\}$ be the respective sets of Blue and Red Agents. Each Blue Agent $i \in \mathcal{B}$ has a frequency $\omega_{i}$ and phase $\beta_{i}$, and similarly each Red Agent $j \in \mathcal{R}$ has frequency $\nu_{j}$ and phase $\rho_{j}$. The Blue Agents are connected to each other symmetrically (which may be relaxed) via the $N \times N$ adjacency matrix $B$ and Red Agents via the $M \times M$ matrix $R$. The $N \times M$ matrix $A$ represents the unidirectional external links from Blue to Red Agents. Figure 1 visualises one possible configuration. Finally, the $\zeta_{B}, \zeta_{R}, \zeta_{B R}$, and $\zeta_{R B}$, are 
the relevant coupling constants. The resulting Boyd-Kuramoto model is

$$
\begin{aligned}
& \frac{d \beta_{i}}{d t}=\omega_{i}-\zeta_{B} \sum_{j \in \mathcal{B}} B_{i j} \sin \left(\beta_{i}-\beta_{j}\right)-\zeta_{B R} \sum_{j \in \mathcal{R}} A_{i j} \sin \left(\beta_{i}-\rho_{j}-\phi\right), \quad i \in \mathcal{B} \\
& \frac{d \rho_{i}}{d t}=\nu_{i}-\zeta_{R} \sum_{i \in \mathcal{R}} R_{i j} \sin \left(\rho_{i}-\rho_{j}\right)-\zeta_{R B} \sum_{j \in \mathcal{B}} A_{i j}^{T} \sin \left(\rho_{i}-\beta_{j}-\psi\right), \quad i \in \mathcal{R},
\end{aligned}
$$

where $d / d t$ is the time derivative, $(\cdot)^{T}$ is the transpose operator, and $\phi$ and $\psi$ are the phase lags (frustrations) [8]. These two phase parameters capture the essence of Boyd's proposal that advantage is gained by one side over the other insofar as the coupled dynamics enable the realisation of one side being ahead of the other by the desired amount: $\phi$ for Blue, and $\psi$ for Red.

In general, the set of nonlinear equations (1) can only be solved numerically and they may exhibit complex and chaotic dynamics. Let

$$
C_{B}:=\frac{1}{N} \sum_{i \in \mathcal{B}} \beta_{i} \text { and } C_{R}:=\frac{1}{M} \sum_{i \in \mathcal{R}} \rho_{i}
$$

be the respective centroids of the Blue and Red Agents' phases. If the respective populations are phase locked internally, i.e. $\beta_{i} \approx \beta_{j}, \forall i, j \in \mathcal{B}$ and $\rho_{i} \approx \rho_{j}, \forall i, j \in \mathcal{R}$, then the difference between the centroids can be defined in a time-invariant manner

$$
\Delta:=C_{B}-C_{R},
$$

where $C_{B}$ and $C_{R}$ are defined in (3). Having a time-invariant, non-zero $\Delta$ can be interpreted as external phase locking between populations. Finally, the degree of synchronisation within a given population is captured via the (local) Kuramoto Order parameters,

$$
O_{B}:=\frac{1}{N}\left|\sum_{i \in \mathcal{B}} e^{j \beta_{i}}\right| \text { and } O_{R}:=\frac{1}{M}\left|\sum_{i \in \mathcal{R}} e^{j \rho_{i}}\right|,
$$

where $j$ denotes the value $\sqrt{-1}$.

\section{Game-Theoretic Formulation}

The adversarial nature of the Blue versus Red interaction and the associated strategic decisions immediately motivate a game-theoretic perspective to the Boyd-Kuramoto model presented in the previous section. Given the novelty of combining game theory with the Boyd-Kuramoto model and as a starting point, a static, 2-Player, nonzero-sum, strategic (non-cooperative) game [3] is formulated next.

The two players of the game constitute the player set $\mathcal{P}:=\{$ Red, Blue $\}$ representing the respective set of networked agent populations $\mathcal{R}, \mathcal{B}$ along with their adjacency matrices $R, B$. The cyclic decision process of each individual agents is described by the respective Boyd (OODA) cycle as described in Kuramoto equations (1). In the game, the players Blue and Red decide on their lead/lag targets $\phi \in[0, \pi]$ and $\psi \in[0, \pi]$, 
respectively. The outcome of the player decisions are captured by the pair of utility functions $U_{B}(\phi, \psi)$ and $U_{R}(\phi, \psi)$, which will be defined later. The resulting game is formally defined by the tuple $\mathcal{G}:=<\mathcal{P},(\phi, \psi),\left(U_{B}, U_{R}\right)>$.

In this game, once the players decide on their respective targets $\phi$ and $\psi$, the BoydKuramoto model (1) is computed numerically over a fixed time-horizon. It is important to note that the equations may not converge to a point or even a limit cycle in certain cases, and may exhibit chaotic behaviour. To capture the existence or the lack of internal phase locking in agent populations, the order parameters in (5) are used. If $O_{B}$ or $O_{R}$ is larger than a threshold value, e.g. 0.9, then an approximate internal phase lock is said to be achieved [8], which is consistent with numerical observations of phase trajectories. Once the players have internal phase locking, then the difference (4) between centroids (3) becomes time-invariant and meaningful. Note that, if there is no internal phase locking even for one player (population), then the phases of agents of that population are time-varying. In that case, it is impossible to talk about a meaningful phase difference $\Delta$ between the Red and Blue centroids.

In light of the discussion above, one possible set of utility functions for the players are

$$
U_{B}(\phi, \psi)= \begin{cases}-(\Delta(T)-\phi)^{2} & , \text { if } O_{B}(T)>0.9, O_{R}(T)>0.9 \\ -100 & , \text { otherwise }\end{cases}
$$

and

$$
U_{R}(\phi, \psi)= \begin{cases}-(-\Delta(T)-\psi)^{2} & , \text { if } O_{B}(T)>0.9, O_{R}(T)>0.9 \\ -100 & , \text { otherwise }\end{cases}
$$

Here, $T$ denotes the finite time horizon over which the Boyd-Kuramoto model (1) is solved numerically, and $O_{B}(T)$ and $O_{R}(T)$ are calculated using Kuramoto model average results over $[T / 4, T]$. The value -100 is chosen as a large negative value to express undesirability of outcomes when $O_{B}(T)<0.9$ or $O_{R}(T)<0.9$, i.e. there is no tangible internal phase locking in Blue or Red player agent populations.

Definition 1. A specific Boyd-Kuramoto Game $\mathcal{G}$ is played between the two adversarial Players $\mathcal{P}:=\{$ Red, Blue $\}$. Each player represents a set of networked agent populations with their decision cycles described by the Boyd-Kuramoto model in (1), which is computed over the time horizon $[0, T]$. The players Blue and Red decide on their lead/lag targets $\phi \in[0, \pi]$ and $\psi \in[0, \pi]$. The outcome of the player decisions are captured by the pair of utility functions $U_{B}(\phi, \psi)$ and $U_{R}(\phi, \psi)$ given in (6) and (7), respectively. Then, the game is defined by the tuple $\mathcal{G}:=<\mathcal{P},(\phi, \psi),\left(U_{B}, U_{R}\right)>$

The Nash Equilibrium (NE) is used in this paper as the main game solution concept. A Nash Equilibrium is formally defined as the set of player strategies and associated utilities, where no player can gain by deviating from the NE when all other players play their own NE strategies. It corresponds to a fixed point as well as the intersection point of the best responses of players [3]. It is worth noting that bi-matrix games always have a solution in mixed strategies, corresponding to a probability distribution over the actions (pure strategies) [3]. 


\section{Numerical Analysis of the Boyd-Kuramoto Game}

\subsection{Properties of the Underlying Boyd-Kuramoto Model}

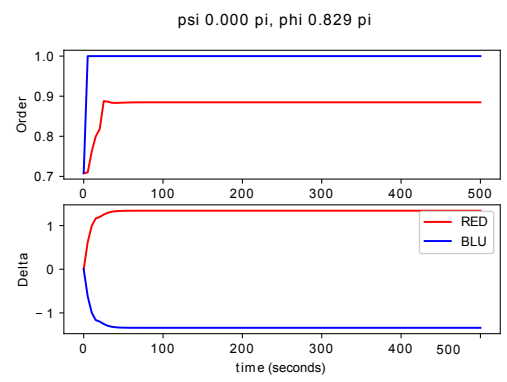

(a) $(\psi, \phi)=(0,0.829 \pi)$

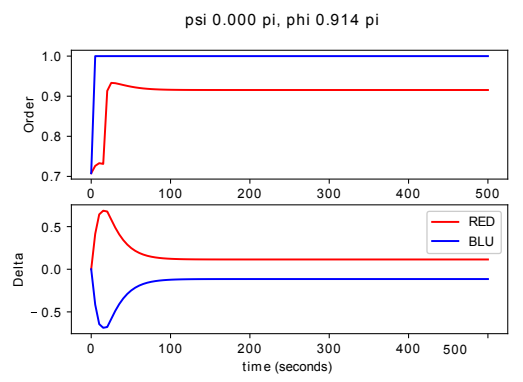

(b) $(\psi, \phi)=(0,0.914 \pi)$

Fig. 2: Plots of $\Delta$ and Order for values approaching instability.

Prior to discussing the specific game results, some considerations on the Kuramoto model dynamics are offered. Depending on the pair of values $(\psi, \phi)$, the Kuramoto model presents stable, oscillatory, or chaotic behaviour. An illustrated example of this behaviour around the values $(\psi, \phi)=(0,0.95 \pi)$ is depicted in Figures 2 and 3 using networks, frequency and coupling choices as given in [8].

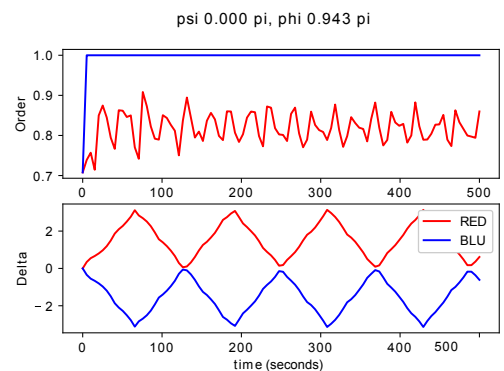

(a) $(\psi, \phi)=(0,0.943 \pi)$

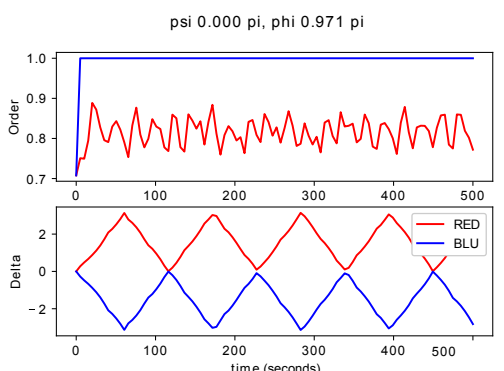

(b) $(\psi, \phi)=(0,0.971 \pi)$

Fig. 3: Plots of $\Delta$ and the Order Parameter for oscillatory/unstable values.

For the parameters in the baseline scenario, the Order Parameter values of the Blue Player are close to full synchronisation, $O_{R} \approx 1$, across all the values of $(\psi, \phi)$ (not illustrated), while the Red Player Order Parameter values are close to synchronisation only in the corner regions of $(\psi, \phi)$, close to $(0,0)$ or $(\pi, \pi)$ as illustrated in Figure 4(a). Figure 4(b) shows the variance of $O_{R}$ and highlights the $(\psi, \phi)$ regions where the system exhibits oscillatory or unstable behaviours as studied in [8]. We observe that the 
region of $(\psi, \phi)$ where the system presents an increased oscillatory behaviour coincides with the region where the Red Order Parameter $O_{R}$ is at a minimum and well below the threshold 0.9 that is penalised in the utility functions of players, $U_{B}(\phi, \psi)$ and $U_{R}(\phi, \psi)$. It constitutes, therefore, a region that both players will tend to avoid in the game.

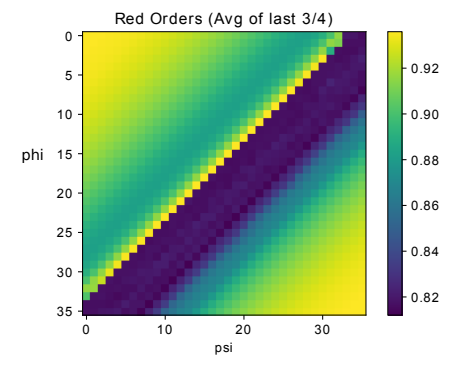

(a) Time average of $O_{R}$



(b) Variance of $O_{R}$

Fig. 4: Red Player Order values and their variance for $(\psi, \phi) \in$ $\left\{0, \frac{\pi}{36}, . ., \frac{n \pi}{36}, . ., \frac{35 \pi}{36}, \pi\right\}$.

\subsection{Nash Equilibrium Solution in Baseline Scenario}

As a baseline scenario for the numerical analysis of the game defined in the previous section, the following parameters have been selected in continuity with the previous studies [6]:

- The Blue network has 21 nodes in a complete 4-ary Tree configuration and the Red network has the same number of (21) randomly connected nodes.

- The interaction network involves the leaves of the Blue Tree (Nodes 6-21) linked to the correspondingly labelled nodes of the random Red network.

- The natural frequencies $\omega$ and $\nu$ are randomly chosen from a $[0,1]$ interval with an average of 0.551329 for the Red agents and 0.503192 for the Blues.

- The coupling constants are $\zeta_{R}=0.5$ for Red and $\zeta_{B}=8$ for Blue, and the crosscoupling constants are $\zeta_{B R}=\zeta_{R B}=0.4$.

The action spaces of the players are discretised to obtain a bi-matrix game. Specifically, each player has 36 strategies available, i.e. the player's respective phase lag targets $\phi$ and $\psi$ are discretised in uniform 36 bins of size $\pi / 36$ within the interval $\{0, \pi\}$. The number 36 is chosen for its divisibility and taking into account computational constraints.

The Kuramoto model (1) is solved for every pair of

$$
(\psi, \phi) \in\left\{0, \frac{\pi}{36}, \ldots, \frac{n \pi}{36}, \ldots, \frac{35 \pi}{36}, \pi\right\} .
$$


The utility functions $U_{B}(\phi, \psi)$ and $U_{R}(\phi, \psi)$ are calculated accordingly using (6) and (7), and provide the entries of the respective utility matrices (Figure 5).

Even though the Boyd-Kuramoto model shows complex dynamic behaviour (as discussed above) depending on the desired phase lags $(\psi, \phi)$, the associated static 2-Player nonzero-sum strategic (non-cooperative) game as formulated in Section 3 admits a pure strategy Nash Equilibrium that is mostly insensitive to the model parameters, except the intrinsic frequencies $\omega_{i}$ and $\nu_{j}$ of the Blue and Red populations.



(a) Red Utility Matrix

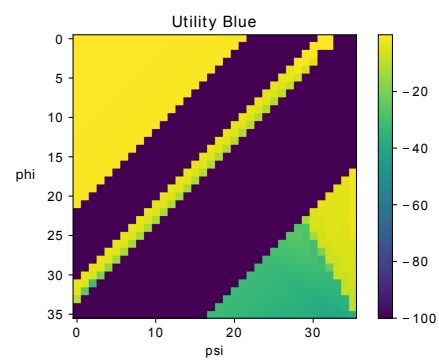

(b) Blue Utility Matrix

Fig. 5: Red and Blue player utilities in the baseline scenario.

For the baseline set of parameters, the player utilities are illustrated in Figure 5. The game admits then a single pure-strategy Nash Equilibrium as shown in Table 1(a). To interpret this result, we first need to appreciate that the maximum reachable utility for a player is equal to zero, that is when the difference (4) between centroids (3) of the system is exactly equal to the desired phase for the player. The calculated NE is therefore stating that the Red player could enforce a very slight phase advantage to the player Blue. In order to increase the resolution of the game discretisation in the area of the utility matrices where the NE emerges, a new simulation has been executed with the same parameters and $(\psi, \phi) \in\left\{0, \frac{\pi}{80}, . ., \frac{n \pi}{80}, . ., \frac{79 \pi}{80}, \frac{\pi}{4}\right\}$. The results, shown in Table 1(b), confirm that Red player can actually enforce a slight lag of $\frac{\pi}{20}$ to the Blue player and gains an advantage at the unique NE of the game. It is also worth noting that this $\mathrm{NE}$ solution is at the same time a Dominant Strategy Equilibrium and the game does not admit any other mixed strategy equilibrium solution.

\section{Analytic Study of the Game Solution}

In [8], the stability properties of the Boyd-Kuramoto model were studied, where it was identified that the fixed point of the dynamical system only depends on the crosscoupling strengths and connectivity between Blue and Red, and the native frequencies of the agents. The sensitivity analysis conducted next in Section 6 indicates that the NE also depends mainly on these parameters, and is relatively insensitive to the network structures and internal couplings of the Blue and Red populations. This suggests an analytic study of the NE is feasible through approximation. 


\begin{tabular}{|c|c|c|}
\hline Player & Strategy & Payoff \\
\hline \hline Red & $\psi=\frac{2 \pi}{36}$ & -0.00033 \\
\hline Blue & $\phi=0$ & -0.02598 \\
\hline
\end{tabular}

(a) NE strategy

\begin{tabular}{|c|c|c|}
\hline Player & Strategy & Payoff \\
\hline \hline Red & $\psi=\frac{1 \pi}{20}$ & 0 \\
\hline Blue & $\phi=0$ & -0.02784 \\
\hline
\end{tabular}

(b) Refined NE

Table 1: Nash Equilibrium strategies of players with refinement (zoom-in at location) in the baseline scenario.

Using the derivation further elaborated in [6], the expression for $\Delta$ may be explicitly computed in the limit where Blue and Red achieve high internal synchronisation, i.e. the origin, as indeed occurs in the region of the observed $\mathrm{NE}\left(\phi^{*}, \psi^{*}\right)=\left(0, \frac{\pi}{20}\right)$ here.

Using the analysis from [8] and [6], we obtain

$$
\begin{array}{r}
\Delta=\varrho+\sin ^{-1}\left(\frac{\mu}{\sqrt{\mathcal{S}^{2}+\mathcal{C}^{2}}}\right) \\
\varrho=\tan ^{-1}(\mathcal{S} / \mathcal{C}) \\
\mathcal{S}=d_{T}^{(B R)}\left(\frac{\zeta_{B R} \sin \phi}{N}-\frac{\zeta_{R B} \sin \psi}{M}\right) \\
\mathcal{C}=d_{T}^{(B R)}\left(\frac{\zeta_{B R} \cos \phi}{N}+\frac{\zeta_{R B} \cos \psi}{M}\right) \\
\mu=\bar{\omega}-\bar{\nu},
\end{array}
$$

where $\Delta$ was defined in (4), $d_{T}^{(B R)}$ is the number of links between Blue and Red, and $\bar{\omega}, \bar{\nu}$ are the mean intrinsic frequencies within the Blue and Red populations. From the baseline scenario chosen in Section $4, d_{T}^{(B R)}=16, \mu=-0.048$.

Substituting the expression above for $\Delta$ in the utility functions for Blue and Red, given in (6), (7), and the parameter choices from Section 4 results in an approximation of player utilities in the stable region, and is shown in Figure 6. The figure also depicts the unconstrained best responses of the players superimposed as lines corresponding to $\partial U_{B} / \partial \phi=\partial U_{R} / \partial \psi=0$, and the numerically obtained unique NE in the baseline scenario. As expected, the NE sits on the line of optima for Red at the edge of the feasible region for Blue, namely $\phi=0$. Moreover, any increase of $\phi$ results in decreasing utility - confirming that Blue cannot improve their outcome. The utility functions of the players at the NE solution are further depicted in Figure 7.

In the region around the origin where the Boyd-Kuramoto dynamics are stable, the expression for $\Delta$ given in (8) can be linearised with respect to $\phi$ and $\psi$ around the point $(0,0)$. In fact, expanding the derivatives $\partial U_{B} / \partial \phi, \partial U_{R} / \partial \psi$ and extracting the roots of $\partial U_{B} / \partial \phi=0=\partial U_{R} / \partial \psi$ leads to the following closed form solution for the Nash 


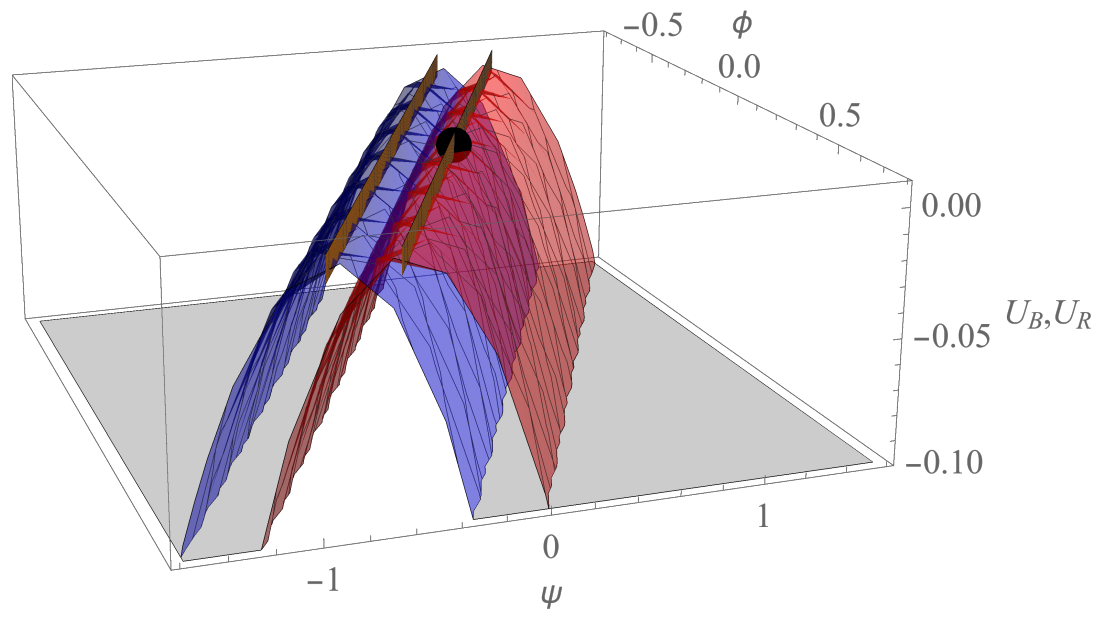

(a) Utility functions of Blue and Red Players around the origin.

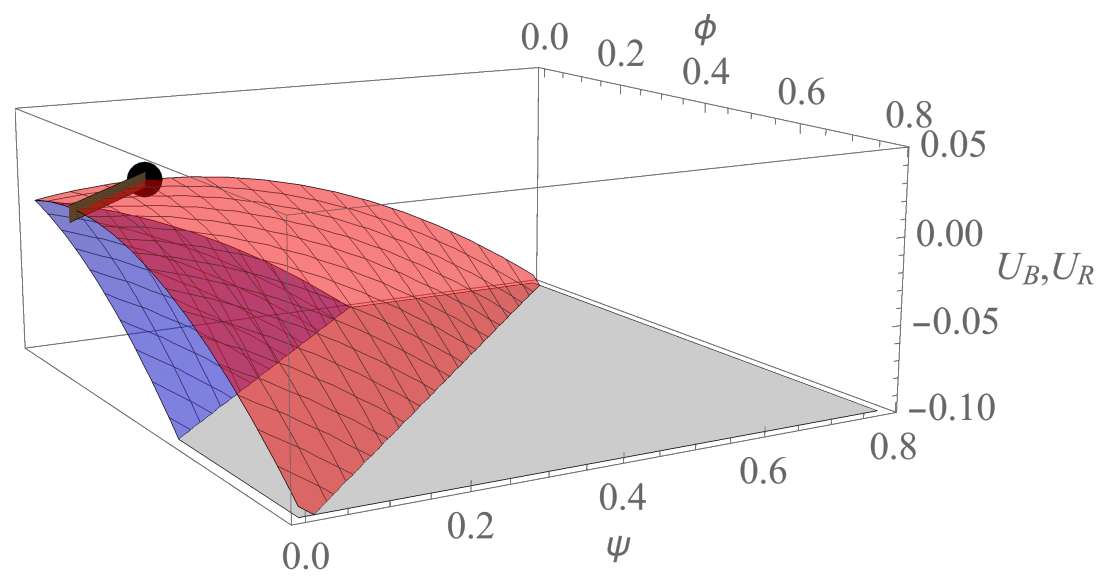

(b) Utility functions over the decision region (positive quadrant) near origin.

Fig. 6: Utility functions of Blue and Red Players calculated using the $\Delta$ expression in (8). The superimposed lines represent the best responses of the players and the black point is the Nash equilibrium. 


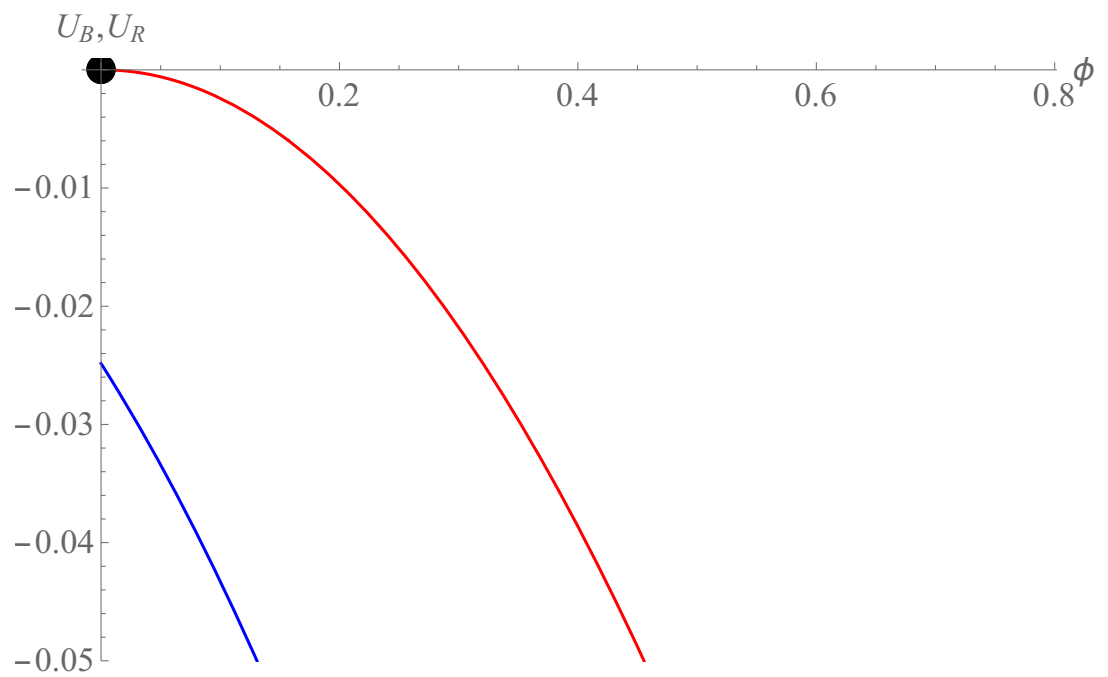

(a) Utility functions of Blue and Red Players at $\psi=\pi / 20$.

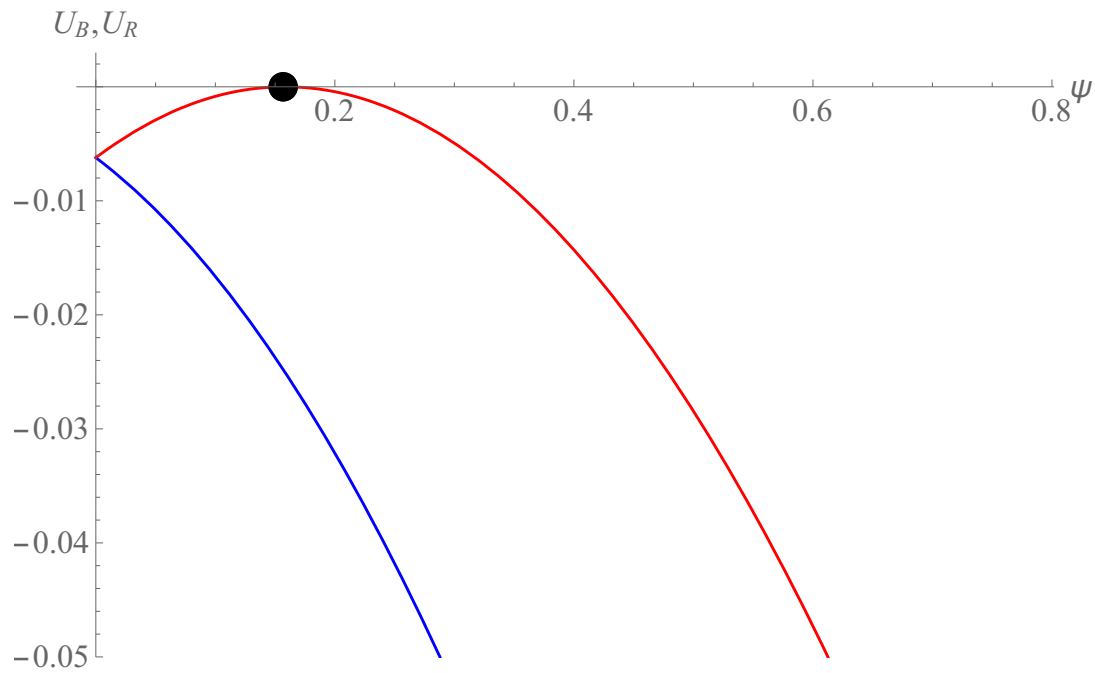

(b) Utility functions of Blue and Red Players at $\phi=0$.

Fig. 7: Utility functions of Blue and Red Players calculated using the $\Delta$ expression in (8) at the Nash Equilibrium, $\left(\phi^{*}, \psi^{*}\right)=\left(0, \frac{\pi}{20}\right)$. 
equilibrium:

$$
\phi^{*}=0, \psi^{*}=-\frac{\left(\zeta_{B R}+\zeta_{R B}\right) \sqrt{d_{T}^{2}\left(\zeta_{B R}+\zeta_{R B}\right)^{2}-N^{2} \mu^{2}} \arcsin \left(\frac{N \mu}{d_{T}\left(\zeta_{B R}+\zeta_{R B}\right)}\right)}{\zeta_{B R} \sqrt{d_{T}^{2}\left(\zeta_{B R}+\zeta_{R B}\right)^{2}-N^{2} \mu^{2}}+N \mu \zeta_{R B} \arcsin \left(\frac{N \mu}{d_{T}\left(\zeta_{B R}+\zeta_{R B}\right)}\right)} .
$$

With the respective parameter values inserted the non-zero phase evaluates to $\psi^{*}=$ 0.156688 , consistent with the previously obtained numerical result, $\pi / 20$. This analytical expression of the NE allows us to observe that the critical factor in generating the asymmetry between Blue and Red is $\mu$, the difference between mean frequencies in the two populations.

\section{Sensitivity Analysis of the Boyd-Kuramoto Game}

The Boyd-Kuramoto Game defined in Section 3 is further analysed numerically in order to gain insights into the solutions as well as their sensitivity to the underlying game parameters. The underlying Boyd-Kuramoto model naturally plays a significant role in the game outcomes. Therefore, four additional scenarios have been explored changing one parameter at a time from the baseline scenario in Section 4. Specifically,

2. Scenario decreases the blue coupling constant $\left(\zeta_{B}\right)$ from 8 to 2 to "loosen" the coupling of the ordered network.

3. Scenario modifies the red network, from random to "hierarchic" (quasi 4-ary matrix).

4. Scenario swaps the natural frequencies $\omega, \nu$ of the Blue and Red player agent populations.

5. Scenario increases the average natural frequency gap between the Blue and Red populations.

\subsection{Scenario 2 - Smaller Blue Player Coupling Constant}

The blue coupling constant $\zeta_{B}$ is decreased to 2 from 8 where all the other parameters remain identical to the baseline scenario. Lowering the blue coupling constant should loosen the tightness of the blue network. In this scenario, the same Nash Equilibrium strategies as the baseline scenario are obtained (Table 2) with a slightly better pay-off for the Red player and worse for the Blue. A possible interpretation is that the red player can enforce slightly more easily the phase gap to a less rigid blue network. It is to be noted that in this scenario, the dynamics of the Boyd-Kuramoto model are very similar to the baseline scenario in terms of pattern and oscillatory behaviour.

\subsection{Scenario 3 - Hierarchical Red Network}

The network matrix of Red player is modified from random to a hierarchical similar to the one of the Blue player and all other parameters are reset to the baseline. In this scenario, the same Nash Equilibrium as per the baseline scenario is obtained (Table 


\begin{tabular}{|c|c|c|}
\hline Player & Strategy & Payoff \\
\hline \hline Red & $\psi=\frac{2 \pi}{36}$ & -0.00001 \\
\hline Blue & $\phi=0$ & -0.03018 \\
\hline
\end{tabular}

Table 2: NE in Scenario 2 with a smaller blue coupling constant of $\zeta_{B}=2$.

3) with comparable player pay-offs. Apparently, with the selected utility function, the structure of the player's network has a very limited influence on the Nash Equilibrium of the game. This can be attributed to the static nature of the game and the underlying time-scale separation between the Kuramoto model dynamics and game formulation. In other words, the game uses an averaged outcome of the Kuramoto model, and hence, the influence of the player networks on the game outcomes diminishes. Note that, similar to Scenario 2, the dynamics of the Boyd-Kuramoto model are very similar to the baseline scenario in terms of pattern and oscillatory behaviour.

\begin{tabular}{|c|c|c|}
\hline Player & Strategy & Payoff \\
\hline \hline Red & $\psi=\frac{2 \pi}{36}$ & -0.00011 \\
\hline Blue & $\phi=0$ & -0.02846 \\
\hline
\end{tabular}

Table 3: NE in Scenario 3 with hierarchical Red network

\subsection{Scenario 4 - Swapping Red and Blue Player Population's Natural Frequencies}

The natural frequencies of Blue player population $\omega$ are swapped with those of the Red player population $\nu$ in order to investigate the impact of natural frequencies on game outcomes. In the baseline scenario, those frequencies are not symmetric and randomly selected from an interval $[0,1]$ with different means: mean $(\omega)=0.5032$ and $\operatorname{mean}(\nu)=0.5513$. The natural frequencies can be interpreted as an input to the Kuramoto system (1).

As a first observation after solving the Boyd-Kuramoto model for this scenario, we realise that the the system dynamics are significantly influenced by the change. While the blue orders are still close to full synchronisation $\left(O_{R} \approx 1\right)$ across all the values of $(\psi, \phi)$ (not illustrated) and the red orders are still close to synchronisation only in the corner regions, we note that the region of $(\psi, \phi)$ for which the system presents oscillatory or unstable properties is displaced compared to the anti-diagonal and with a significant increase of variance all around the anti-diagonal as illustrated in Figure 8 compared to the baseline scenario (Figure 4). Figure 9 shows the utilities for Red and Blue players, which also reflects similar displacement compared to the baseline scenario (Figure 5). 


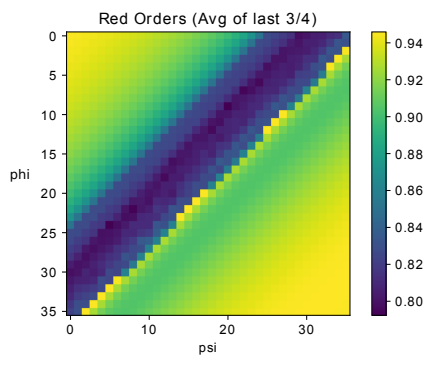

(a) Time average of $O_{R}$

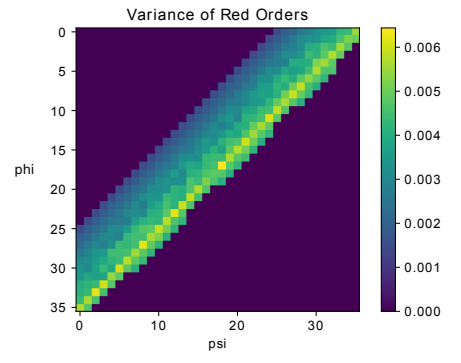

(b) Variance of $O_{R}$

Fig. 8: Red Orders and their variance for $(\psi, \phi) \in\left\{0, \frac{\pi}{36}, . ., \frac{n \pi}{36}, . ., \frac{35 \pi}{36}, \pi\right\}$

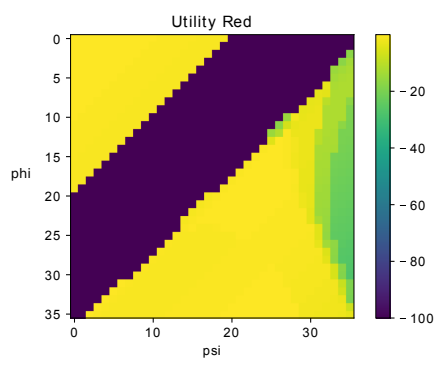

(a) Red Utility

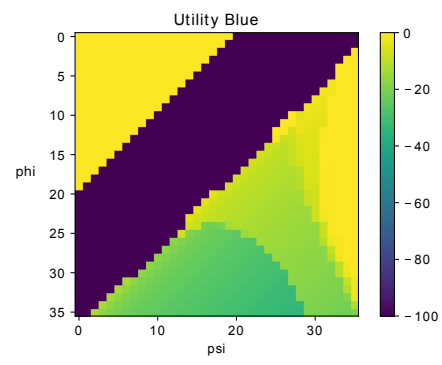

(b) Blue Utility

Fig. 9: Red and Blue player utility matrices with swapped natural frequencies. 
As an important second observation, when solving the game for Nash Equilibrium in this scenario, we note an inversion of winner in the game as illustrated in Table 4. The calculated Nash Equilibrium shows now the Blue player being able to enforce on the Red player a small phase gap of $\frac{3 \pi}{36}$ with a symmetric pay-off compared to the baseline scenario. Considering that the swap of frequencies between blue and red is actually reversing the asymmetry of frequencies between players, it appears that the asymmetry of the natural frequencies is playing an important role. Specifically, the 'faster' player in average can enforce a small phase lag to the 'slower' player.

\begin{tabular}{|c|c|c|}
\hline Player & Strategy & Payoff \\
\hline \hline Red & $\psi=0$ & -0.06512 \\
\hline Blue & $\phi=\frac{3 \pi}{36}$ & -0.00019 \\
\hline
\end{tabular}

Table 4: Nash Equilibrium - scenario 4 - swapped frequencies

\subsection{Scenario 5 - Increased Natural Frequency Gap between Players}

In this scenario, the original frequency gap between red and blue player is doubled by maintaining the original blue frequencies as per baseline mean $\left(\omega_{i}\right)=0.5032$ and shifting the red frequencies further so that mean $\left(\nu_{j}\right)=0.5994$ from 0.5513 as per baseline value. Similar to the previous scenario, we observe that the system dynamic is significantly influenced by that change. The blue orders are still close to full synchronisation $\left(O_{R} \approx 1\right)$ across all the values of $(\psi, \phi)$ (not illustrated) and the red orders are still close to synchronisation only in the corner regions. We note that the region of $(\psi, \phi)$, for which the system presents oscillatory or unstable properties, shows a significant increase in variance all around the anti-diagonal as illustrated in Figure 10, when compared to the baseline scenario in Figure 4. Solving the game for Nash Equilibrium, we observe that the increase of Red player natural frequency allows it to slightly increase the imposed phase lag to $\frac{3 \pi}{36}$ compared to $\frac{2 \pi}{36}$ of the baseline scenario (Table 5).

\begin{tabular}{|c|c|c|}
\hline Player & Strategy & Payoff \\
\hline \hline Red & $\psi=\frac{3 \pi}{36}$ & -0.00049 \\
\hline Blue & $\phi=0$ & -0.11346 \\
\hline
\end{tabular}

Table 5: Nash Equilibrium in Scenario 5 with increased gap of frequencies 


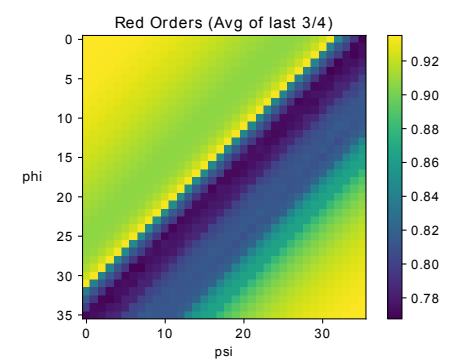

(a) Time average of $O_{R}$

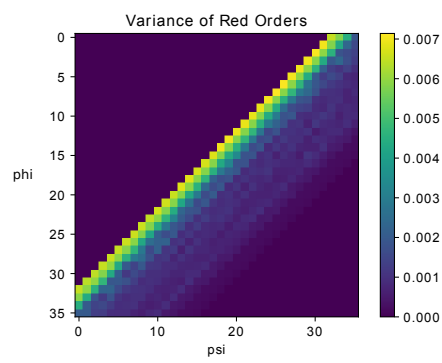

(b) Variance of $O_{R}$

Fig. 10: Red Orders and their variance in Scenario 5.

\section{Conclusion}

The initial results in this paper show that combining a game-theoretic approach with the Boyd-Kuramoto model is a fertile research direction for better understanding of strategic decisions in complex and competitive/adversarial systems.

The Boyd-Kuramoto Game studied as a baseline scenario in this report admits a unique pure Nash Equilibrium solution that has interesting implications. The subsequent sensitivity analysis provides additional insights. It is observed numerically that many parameters of the Kuramoto model affect the NE solution of the current game formulation much less than initially stipulated. This is arguably due to the time-scale separation between the underlying Kuramoto model and the static game built on top of it. While these parameters play an important role in the Boyd-Kuramoto dynamics, they do not directly affect (stable) stationary outputs. We expect this situation may change in dynamic game formulations, which may encourage more dynamic decisions than formulated in this paper. In terms of the dynamical system, it means that the behaviour of fluctuations - which are known to depend sensitively on the network structures [6] will have an impact on the game dynamics. Numerical simulations also indicate that the natural frequencies of the agents have a significant impact on NE outcomes of the game. Again, this can be attributed to the fact that the natural frequencies have a direct impact on the underlying Kuramoto model's stationary solutions. From a decision-making perspective, this may be interpreted as investment in individual agents may pay-off more in certain cases than their organisational properties. All of these hypotheses are supported by the analytic solution obtained by linearisation around the origin. The analysis makes it explicitly clear that the static game outcomes depend on the cross-couplings and numbers of connections, and the asymmetry between mean frequencies in the two adversary populations.

There are multiple potentially promising future directions including investigation of different utility functions and dynamic game formulations that allow the players to change their decisions during the evolution of the underlying Boyd-Kuramoto models. 


\section{Acknowledgements}

This research was supported through the Australian Defence Science and Technology Group's Strategic Research Initiative 'Modelling Complex Warfighting'.

\section{References}

1. Acebrón, J.A., Bonilla, L.L., Pérez Vicente, C.J., Ritort, F., Spigler, R.: The Kuramoto model: A simple paradigm for synchronization phenomena. Rev. Mod. Phys. 77, 137-185 (Apr 2005), https://link.aps.org/doi/10.1103/RevModPhys.77.137

2. Alpcan, T., Başar, T.: Network Security: A Decision and Game Theoretic Approach. Cambridge University Press (2011)

3. Başar, T., Olsder, G.J.: Dynamic Noncooperative Game Theory. Philadelphia, PA: SIAM, 2nd edn. (1999)

4. Boyd, J.: A discourse on winning and losing. Maxwell Air Force Base, AL: Air University Library Document No. M-U 43947 (1987)

5. E. Montbrio, J. Kurths, B.B.: Synchronization of two interacting populations of oscillators. Phys.Rev. E 70 (2004)

6. Holder, A.B., Zuparic, M.L., Kalloniatis, A.C.: Gaussian noise and the two-network frustrated Kuramoto model. Physica D: Nonlinear Phenomena 341, 10-32 (2017), http: // www.sciencedirect.com/science/article/pii/S016727891630152X

7. Kalloniatis, A.C.: On the 'Boyd-Kuramoto Model': Emergence in a Mathematical Model for Adversary C2 Systems (2012)

8. Kalloniatis, A.C., Zuparic, M.L.: Fixed points and stability in the two-network frustrated Kuramoto model. Physica A: Statistical Mechanics and its Applications 447, 21-35 (2016), http: / / www. sciencedirect.com/science/article/pii/ s0378437115010092

9. Kuramoto, Y.: Chemical oscillations, waves and turbulence (1984) 


\section{University Library}

\section{- M M I N E R VA A gateway to Melbourne's research publications}

Minerva Access is the Institutional Repository of The University of Melbourne

Author/s:

Demazy, A;Kalloniatis, A;Alpcan, T

Title:

A game-theoretic analysis of the adversarial boyd-kuramoto model

Date:

2018-01-01

Citation:

Demazy, A., Kalloniatis, A. \& Alpcan, T. (2018). A game-theoretic analysis of the adversarial boyd-kuramoto model. Bushnell, L (Ed.) Poovendran, R (Ed.) Basar, T (Ed.) Decision and Game Theory for Security 9th International Conference, GameSec 2018, 11199 LNCS, pp.248-264. SpringerLink. https://doi.org/10.1007/978-3-030-01554-1_14.

Persistent Link:

http://hdl.handle.net/11343/241554 